Preface

\title{
Recent Advances in Thrombosis and Hemostasis-Part II
}

\author{
Sam Schulman, MD, $\mathrm{PhD}^{1,2}$ Md. Shahidul Islam, MD, $\mathrm{PhD}^{3,4}$ \\ 1 Department of Medicine, Thrombosis and Atherosclerosis Research \\ Institute, McMaster University, Hamilton, Ontario, Canada \\ 2 Department of Medicine, Karolinska Institutet, Stockholm, Sweden \\ ${ }^{3}$ Department of Clinical Science and Education, Karolinska Institutet, \\ Stockholm, Sweden \\ ${ }^{4}$ Department of Emergency Care and Internal Medicine, Uppsala \\ University Hospital, Uppsala University, Uppsala, Sweden
}

Semin Thromb Hemost 2017;43:809-810.

Venous thromboembolism leads the clinician mainly to think of deep vein thrombosis in the leg and pulmonary embolism. There are also unusual sites for thrombosis that the consultant has to deal with not infrequently. The cause of venous thrombosis is typically a trigger from surgery, trauma, immobilization, or cancer, and if nothing of this kind is identified it is called "unprovoked." There are, however, unusual situations where venous thromboembolism occurs, such as vascular malformations or rare inflammatory or autoimmune disorders. The mechanism behind thrombus generation is not always clear, although in case of tissue injury the extrinsic pathway is easily implicated. There may, however, be other mechanisms involved, for example, when venous stasis results in hypoxia in the stagnant blood behind the venous valves or in inflammation, perhaps resulting in neutrophil extracellular trap formation or NETosis. The pathophysiology may include other alternatives as well. The treatment of venous thromboembolism has been studied in many randomized clinical trials for the adult population but hardly at all in children. An often-debated topic is the duration of anticoagulation after venous thromboembolism, and although the risk of recurrence probably never goes down to zero, many physicians feel reluctant to treat indefinitely due to the risk of bleeding. Prediction rules might give some support for decisions in this situation.

Many of these topics are dealt with in this second part of the "Recent advances in thrombosis and hemostasis" issue theme. We hope that the readers of Seminars in Thrombosis and Hemostasis will find the articles interesting and also helpful for their clinical practice. Half of the topics were discussed by well-known scientists in the field in association with a congregation in Stockholm, Sweden, in November 2016, itself the subject of a separate meeting report in the current issue of the journal. ${ }^{1}$ The remaining contributions were selected from other submissions accepted for publication over recent past months.

Address for correspondence Sam Schulman, MD, PhD, Thrombosis Service, HHS-General Hospital, 237 Barton Street East, Hamilton, ON L8L 2X2, Canada (e-mail: schulms@mcmaster.ca).
Naudin et al begin this issue and provide us with a review of the role of polyphosphate (polyP), a potent contact activator, and factor XII in the generation of arterial or venous thrombosis mediated by platelets. ${ }^{2}$ This pathway is in fact already being explored as a target for drugs to prevent or treat thrombosis. In addition, polyP activates the bradykininmediated pathway to inflammation. We will surely learn much more about polyP in the next few years.

A very rare cause of deep vein thrombosis is agenesis of the inferior vena cava, but its prevalence might be underestimated due to inadequate diagnosis. Tufano et al have authored a comprehensive description of this condition based on local experience and a literature review. ${ }^{3}$ It is particularly important to keep this vascular malformation in mind when deep vein thrombosis occurs in a young patient. Venous thrombosis in the distal leg veins, in other words below the trifurcation, is not captured with the typical compression ultrasound examination performed in most hospitals. This is not considered a problem because many consider distal deep vein thrombosis as clinically unimportant and not necessary to treat. Lim et al have performed a meta-analysis of the randomized controlled trials and the cohort studies published. ${ }^{4}$ They find that this common perception might be wrong and that there probably are benefits without increased risk of harm to diagnose and treat these patients.

Although the diagnosis of atrial fibrillation almost always justifies anticoagulant therapy, it is often not realized that there can be concomitant pulmonary embolism, which sometimes might change the treatment strategy. Bikdeli et al present a systematic review of the coexistence of these entities. ${ }^{5}$ They discuss which one of these could have occurred first, with the alternate pathology as a consequence, the epidemiology, the prognostic significance, and the therapeutic options. Interestingly, a clinical prediction rule for risk of stroke in atrial fibrillation $\left(\mathrm{CHA}_{2} \mathrm{DS}_{2}\right.$-VASc) seems to predict the mortality in pulmonary embolism without coexisting atrial fibrillation. Continuing on this theme, Tang et al used their historic hospital

Copyright $\odot 2017$ by Thieme Medical Publishers, Inc., 333 Seventh Avenue, New York, NY 10001, USA. Tel: +1(212) 584-4662.
DOI https://doi.org/ 10.1055/s-0037-1605566. ISSN 0094-6176.
Issue Theme Recent Advances in Thrombosis and HemostasisPart II; Guest Editors: Sam Schulman, MD, PhD, and Md. Shahidul Islam, MD, PhD. 
population of over 4,000 patients with pulmonary embolism to identify 305 patients with concomitant atrial fibrillation and then explore the predictive value for in-hospital outcome with the $\mathrm{CHADS}_{2}$ score, the $\mathrm{CHA}_{2} \mathrm{DS}_{2}$-VASc score, and the pulmonary embolism severity index (PESI). ${ }^{6}$ Although PESI had the best predictive value, addition of the $\mathrm{CHADS}_{2}$ score seemed to improve it further. Finally, Alatri et al present an analysis of yet another prediction rule, the modified Ottawa score for hospitalized patients with cancer and venous thromboembolism. ${ }^{7}$ Although the score did not turn out to be useful for the prediction of recurrent venous thromboembolism, it indicated risk of early mortality.

Thrombosis in neonatal patients is almost always provoked by obvious risk factors, such as central venous catheters in combination with serious pathological conditions. Radulescu informs us here of the epidemiology of venous thromboembolism in the pediatric population and reviews the large number of therapeutic options available, finalizing with a discussion on the duration of anticoagulation. ${ }^{8}$

The main risk of antithrombotic therapy, in young and old, is hemorrhage. The most common major hemorrhage is gastrointestinal and the most feared, due to high mortality and frequent residual neurologic impairment, is intracranial bleeding. Schulman reviews the management of major bleeding, which differs between the anticoagulants. ${ }^{9}$ The non-vitamin $\mathrm{K}$ antagonist anticoagulants, often abbreviated NOACs, are progressively gaining market shares from warfarin and its derivatives and it is useful to be aware of the currently available options to reverse bleeding, should this occur. Once the hemorrhagic complication has been controlled, the balance between risk for thromboembolic events and the risk of recurrent hemorrhage has to be weighed. Studies that support such a decision after intracranial or gastrointestinal hemorrhage are also discussed here.

The concluding article in this theme issue may seem a bit off topic, focusing on endovascular treatment of stroke, that is, intervention on the arterial side to remove clots from small vessels in the brain. Until recently, this was very uncertain territory and intravenous thrombolysis was considered the optimal choice unless contraindicated. Cagnap and Smith are here supporting the recommendation for the endovascular approach in eligible patients based on results from recent trials. ${ }^{10}$ This treatment modality requires expertise but also changes in the logistics for the emergency management of patients with ischemic stroke.

As mentioned, we are also tying up any remaining loose ends of the first two parts of the current issue theme with a brief report by the guest editors, but primarily from the meeting organizer, Md. Shahidul Islam, of the 93rd Berzelius Symposium, the meeting where most of the contained topics were discussed. $^{1}$

We are pleased to publish this compilation with the conviction that many readers will find the reviews, as well as the original data presented in some papers, both interesting and helpful in their decisions as consultants or most responsible physicians. These articles complement the ones in Part I of this theme issue, published in November 2016. ${ }^{11}$ The letter to the editor at the end of this issue, ${ }^{12}$ and the response from the original authors, ${ }^{13}$ pertains to one of those articles in Part I. ${ }^{14}$

\section{References}

1 Islam MS, Schulman S. Berzelius Symposium 93: International Conference on Thrombosis and Embolism. Semin Thromb Hemost 2017;43(08):811-813

2 Naudin C, Burillo E, Blankenberg S, Butler L, Renné T. Factor XII contact activation. Semin Thromb Hemost 2017;43(08):814-826

3 Tufano A, Cannavacciuolo F, Gianno A, et al. Inferior vena cava agenesis and deep vein thrombosis in the young: a review of the literature and local experience. Semin Thromb Hemost 2017;43 (08):827-835

4 Lim MS, Ariyariah A, Oldmeadow C, Hall A, Enjeti AK. A systematic review and meta-analysis comparing anticoagulation versus no anticoagulation and shorter versus longer duration of anticoagulation for treatment of isolated distal DVT. Semin Thromb Hemost 2017;43(08):836-848

5 Bikdeli B, Ziki MD, Lip GY. Pulmonary embolism and atrial fibrillation: two sides of the same coin? A systematic review. Semin Thromb Hemost 2017;43(08):849-863

6 Tang R-B, Xu Z-Y, Avula UM, et al. Risk stratification for acute pulmonary embolism in patients with atrial fibrillation: role of $\mathrm{CHADS}_{2}$ score. Semin Thromb Hemost 2017;43(08):864-870

7 Alatri A, Mazzolai L, Kucher N, et al. The modified Ottawa score and clinical events in hospitalized patients with cancer-associated thrombosis from the Swiss VTE registry (SWIVTER). Semin Thromb Hemost 2017;43(08):871-876

8 Radulescu VC. Anticoagulation therapy in children. Semin Thromb Hemost 2017;43(08):877-885

9 Schulman S. Bleeding complications and management on anticoagulant therapy. Semin Thromb Hemost 2017;43(08):886-892

10 Cagnap S, Smith WS. Endovascular stroke therapy. Semin Thromb Hemost 2017;43(08):893-901

11 Schulman S, Islam MS. Recent advances in thrombosis and hemostasis - Part I. Semin Thromb Hemost 2016;42(08): 805-807

12 Bajc M, Grüning T. Comment on "state-of-the-art imaging in pulmonary embolism: ventilation/perfusion single-photon emission computed tomography versus computed tomography angiography-controversies, results, and recommendations from a systematic review". Semin Thromb Hemost 2017;43(08): 902-903

13 Hess S, Frary EC, Gerke O, Madsen PH. Response to "comment on state-of-the-art imaging in pulmonary embolism: ventilation/ perfusion single-photon emission computed tomography versus computed tomography angiography-controversies, results, and recommendations from a systematic review". Semin Thromb Hemost 2017;43(08):904-906

14 Hess S, Frary EC, Gerke O, Madsen PH. State-of-the-art imaging in pulmonary embolism: ventilation/perfusion single-photon emission computed tomography versus computed tomography angiography - controversies, results, and recommendations from a systematic review. Semin Thromb Hemost 2016;42(08):833-845 